\title{
Digital Scope on 2D Communication Sheet for Location-Specific Multimedia Service
}

\author{
Bing Zhang ${ }^{1}$, Youiti Kado ${ }^{1}$, Kiyohiko Hattori² and Jiang Yu Zheng ${ }^{3}$ \\ ${ }^{1}$ National Institute of Information and Communication Technologies \\ ${ }^{2}$ University of Electro-Communications \\ Indiana University Purdue University Indianapolis, \\ 1,2Japan \\ ${ }^{3}$ USA
}

\section{Introduction}

In recent years, multimedia data have explosively increased in the web environment for education, investigation and exploration. At the same time, augment reality research has paid a great attention to providing views and contents in a real setting to enhance the perception of environments and spaces. A basic function - position aware display is demanded in such systems for many applications. Examples include finding locationspecific data in a map [1-5], exploring media related to an object [6-7], viewing process at a spot, watching structure and diagnosing a part of human body, simulating various effects in a designed space, etc.

Position indexing has been realized on normal computer screen by reading various input devices. However, such systems are not sufficient for indexing details from a large map or hierarchical maps. The windows showing different media such as a map and a street view are usually displayed separately and it requires some effort to correspond the location between two windows [2]. This design has been generalized to many machine interfaces with large scale where the hand movement is tracked by cameras and the detected position drives scenes on another separate screen. In the tabletop projections, two views with different resolutions and media are combined [8-10] with a projector and sensor systems. In this chapter, we construct a platform on which users can watch details just as a magnificent by touching a special sheet.

To develop a new communication technology that complements wired and wireless communications, a special physical medium has been developed to perform both data and power transmission inside a 2D sheet [11]-[13]. Through a connector put on the top sheet, an electromagnetic (EM) proximity connection is obtained to receive and send the electromagnetic signals, while moving the connector mounted device away from the sheet disconnects the transmission [14]. The size of a sheet can be as wide as several meters and is inexpensive in production. The signal transmission is different from the wireless communication that overwhelms the entire 3D space; it covers a wide surface or on partitioned surfaces to facilitate information transmission and visualization. The sheets can 
be laid on a table, wall, and floor in living and working spaces. By putting a display device onto the sheet, we can look into the information at that position by receiving the signal from a computer transmitted via the sheet.

We design and build a system that can enlarge details at a location or show related media data without directing viewer's focus to separated screens or windows. The system has a function of magnifier or scope to pin-point locations with a small display on a surface. Moreover, because there has no successful example of curved screens that can fit on a curved body for clicking and display, we can then wrap a surface with this two dimensional sheet for signal transmission. By placing a small high-resolution LCD on the sheet, we will be able to see position specific information and media detailed in resolution in contrast to a global map laid on the surface. In this chapter, we introduce the design and implementation of a platform that can look into a $2 \mathrm{D}$ or even 3D space by touching the surface sheet with a small display. Several tasks will also be examined to evaluate the performance of the platform. Figure 1 shows such an idea to magnify details at designated locations of an atlas.

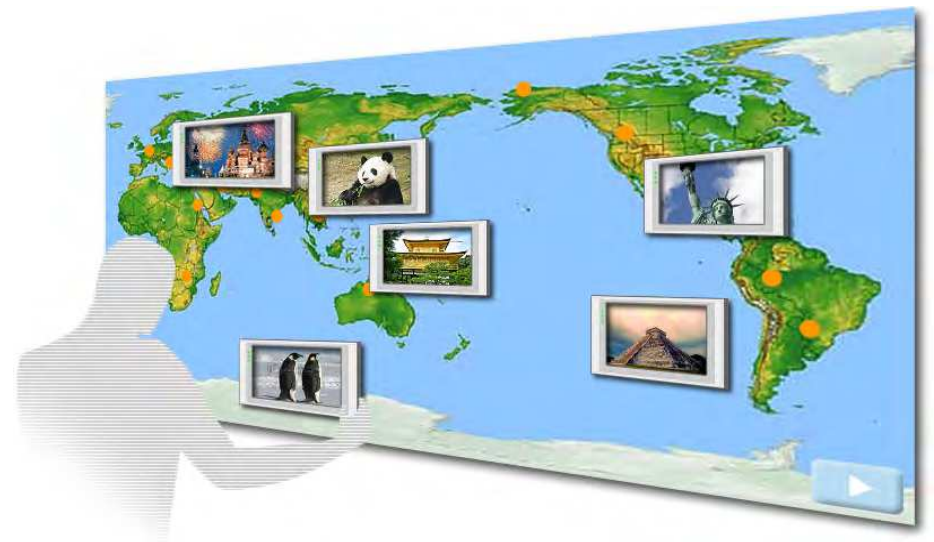

Fig. 1. Digital scope on a communication sheet to view media contents at indicated locations in an atlas.

\section{The sheet as communication medium}

A 2D communication system (2DCS) consists of two components: a sheet and a connector. Figure 2 illustrates the basic structure of the 2D sheet, which contains three layers in order: conductive layer, dielectric layer and mesh conductive layer from the bottom. The conductive fabric is usually copper or aluminum, whereas the dielectric material is polystyrene. With this layered composition, an electromagnetic wave can be confined within the 2D sheet depending on the relatively permittivity of dielectric layer and mesh size of conductive layer. The interval and width of mesh structure are determined from the wave length of the electromagnetic wave. In the case of $2.4 \mathrm{GHz}$ microwave, the interval and width of the stripes in mesh structure are designed to be $7 \mathrm{~mm}$ and $1 \mathrm{~mm}$ respectively, as shown in Fig.2 (Top). The electromagnetic field seeped out from the sheet is called as "evanescent wave" as shown as Fig.2 (Bottom), which spreads only to the vicinity of the sheet. When the connector is brought close to the sheet, the energy is transmitted to the connector through the evanescent wave. 

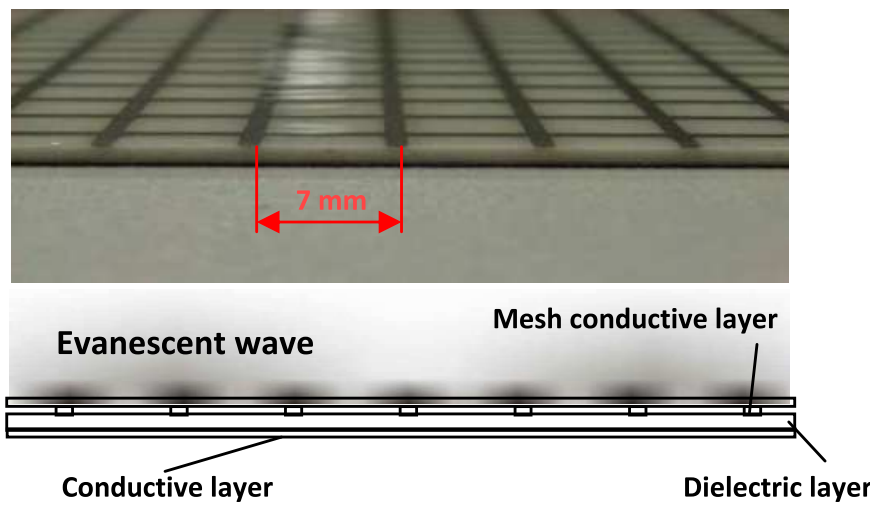

Fig. 2. Two dimensional communication sheet. (Top) 2D sheet construction; (Bottom) The electromagnetic wave seeping out from the 2D sheet.

Meanwhile, the connector is an antenna by which an electromagnetic wave is extracted from or inserted into the 2D sheet. An example of the connector was designed and proposed by Yamahira et al. [14]. The proposed connector consists of metal, dielectric material with the relative permittivity of 10.5, and a subminiature type-A (SMA) connector. The radius of the connector was also determined so that the reflection of the electromagnetic wave from the connector to a cable is minimized at the frequency of $2.4 \mathrm{GHz}$.

Besides the communication, the power can also be supplied through the $2 \mathrm{D}$ communication sheet. A device such as PDA can be put anywhere on the sheet to work without extra wires and batteries, which forms a very flexible system of distributed displays. The sheet can be manufactured in a very low cost affordable to be put on table top, walls, and poster boards. The connector is an antenna by which an electromagnetic wave is extracted from or inserted into the $2 \mathrm{D}$ sheet. The connector is manufactured in a small piece to contact the sheet for receiving and passing signals. Figure 3 shows a set of connector (each piece is $25 \times 70 \times 3 \mathrm{~mm}$ and weights $2 \mathrm{~g}$ ) and a small display (LCD of $800 \times 480$ pixels) that can be put on any position of the communication sheet to receive data and power signals.

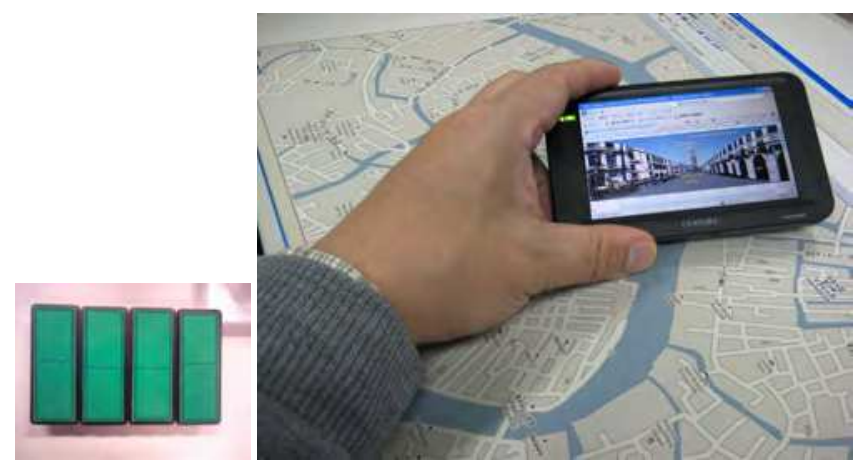

Fig. 3. Digital scope by a lightweight high-resolution display receiving data signal through the $2 \mathrm{D}$ sheet. The connector for the display contacts the sheet to receive the power signal. 


\section{Position detection on communication sheet}

To develop the location-specific multimedia applications for 2D communication system, it is highly important to accurately estimate the position where the display is placed over the sheet. Here, two position identification approaches are examined in our platform: (a) position sensing the arrival time difference between the infrared and ultrasound waves, and (b) position sensing the arrival phase difference between the multiple radio inputs.

\subsection{Positioning by sensing arrival time difference}

The first approach uses infrared and ultrasound technologies. Two kinds of transmitters are adopted to send the infrared and ultrasonic waves, which are mounted on the display. A sensor bar including two kinds of receivers sensing the infrared and ultrasonic waves is set up on one side of the 2D communication sheet. Upon receiving the infrared and ultrasonic signals from transmitters, the distance between display and sensor bar is acquired from the arrival time difference of two waves. Figure 4 shows the mechanism that the relative position of transmitter is calculated from $S_{0}, S_{1}$, and $W$, which are the distances between the transmitter and ultrasonic receiver, transmitter and another ultrasonic receiver, and two ultrasonic receivers, respectively, as follows

$$
\begin{gathered}
y=\frac{s_{0}^{2}-s_{1}^{2}+W^{2}}{2 * W} \\
x=\sqrt{s_{0}^{2}-y^{2}}
\end{gathered}
$$

Through the above Trilateration, the location of the transmitter can be determined.

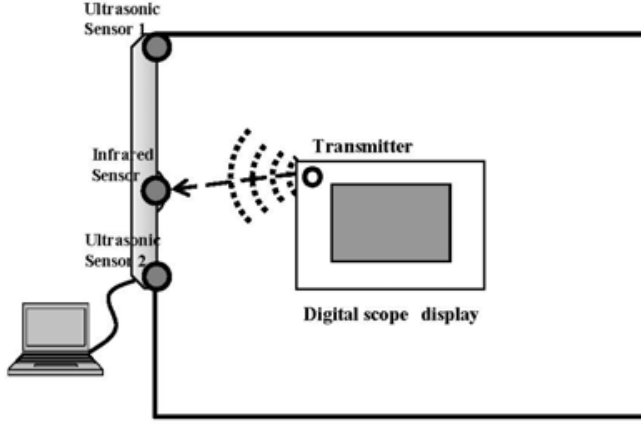

2D sheet

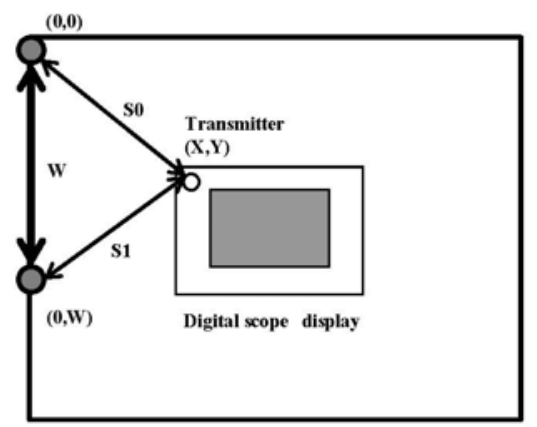

2D sheet

Fig. 4. Acquiring the relative position by the arrival time difference between the infrared and ultrasound waves.

To obtain the orientation of the display in addition to the position, two positioning sets are mounted on two corners of the display. Since the sensor bar cannot simultaneously receive the location information from two positioning sets, a relay switch is built into the display side, as shown in Fig. 5. The relay switch on the display receives the ZigBee signal from the computer via the communication sheet and then controls the turn of the transmitters in sending pulses. The positions of transmitters are linked as a vector that defines the 

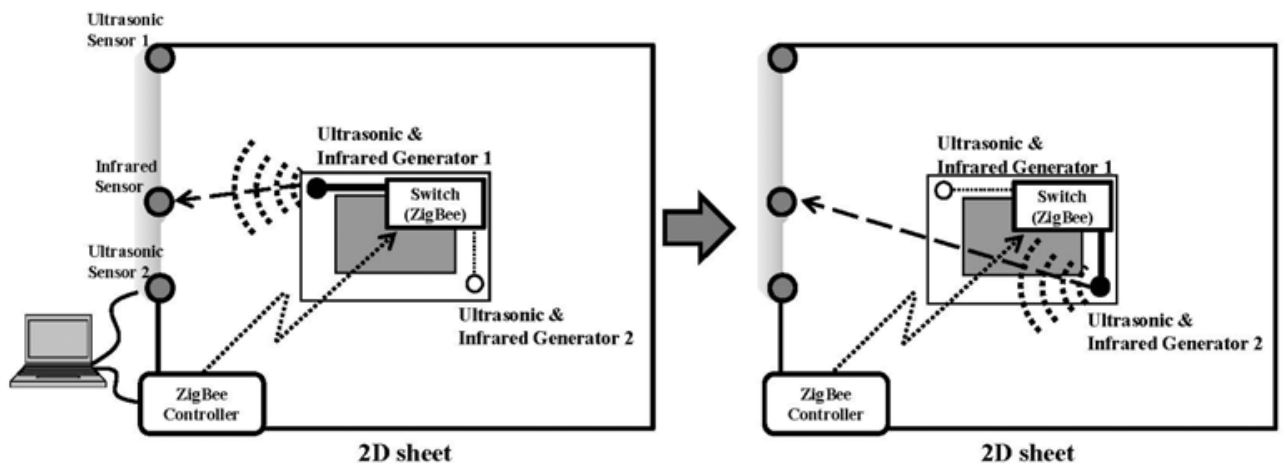

Fig. 5. The signals from two positioning sets are alternatively sent to the receivers by using the wireless Zigbee switch controller. (a) The positioning set 1 is turned on switched by Zigbee controller, (b) The positioning set 2 is turned on.

orientation of the display. The temporal output of the vector provides a trace of orientation moving on top of the communication sheet as depicted in Fig. 6. The drawbacks of this approach are the necessity of the additional sensor devices to generate and sense the infrared and ultrasound waves, as well as the influence from surrounding objects and human bodies, because the reflection of beams from other objects makes the signal noisy when reaching the receivers.

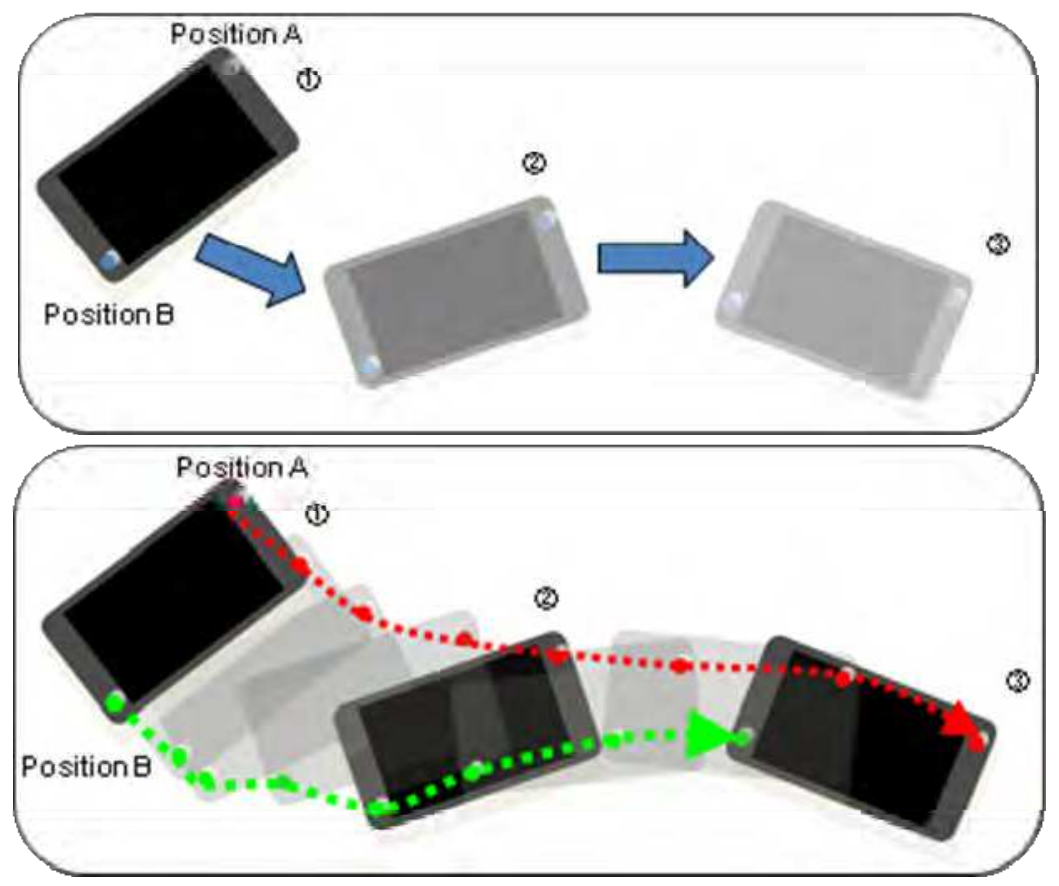

Fig. 6. Obtaining location and orientation of a display device on the 2D sheet. 


\subsection{Positioning by sensing arrival phase difference}

To avoid the interference to the other radio communications performed outside of the sheet, the second approach sends signal within the communication sheet. To aim at a simple system, instead of the utilization of the extra infrared and ultrasound waves mentioned in subsection 3.1, we directly employ the microwaves applied in the 2D communication system for position estimation. We employ a small signal emitter mounted on the scope device that is called target device, and attach the multiple microwave receiving electrodes on the sides of the communication sheet, as shown in Fig. 7. As the microwave runs much faster than the ultrasonic wave, even when the time clock is $3.4 \mathrm{GHz}$ achieved by the chip-scale atomic clock in one of DARPA projects in 2007 [15], the precision of the measured distance computed by the arrival time of radio beacon is about $10 \mathrm{~cm}$. Instead of directly measuring the arrival time of microwave, we here examine another approach in which we measure the phase difference arriving at the different abovementioned electrodes, by transmitting the pilot signal from the target device.

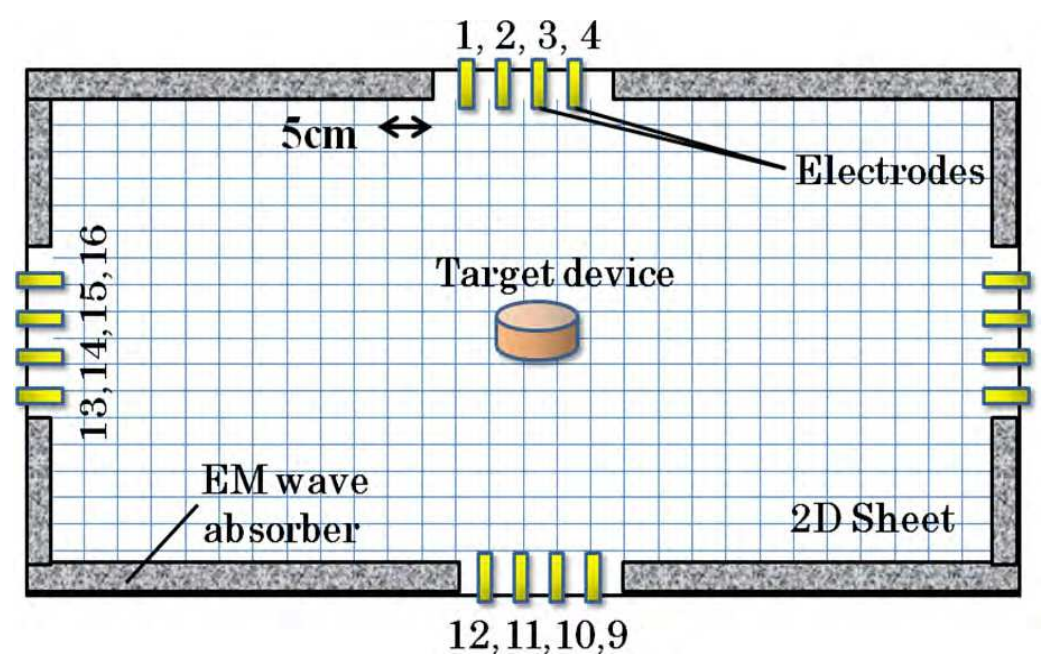

Fig. 7. Positioning of target device using phase difference of electrode array on 2D Communication sheet.

When a sinusoidal wave at radio frequency is transmitted from a targeted device via a coupler on the $2 \mathrm{D}$ sheet, it is propagated through the $2 \mathrm{D}$ sheet as a cylindrical wave. Figure 8 schematically shows how the phase shift and difference of received signal strengths (RSS) occur between two electrodes at different distance $d_{1}$ and $d_{2}$ from the target device on a 2D sheet under the ideal condition. The waveform in the $2 \mathrm{D}$ sheet can be approximated by the following equation.

$$
\mathrm{f}=\mathrm{A}(\mathrm{d}) \mathrm{e}^{\mathrm{i}(\mathrm{kd}-\omega \mathrm{t}+\varnothing)}
$$

Where $d$ is the distance from the target, $A(d)$ is amplitude corresponding to the distance, $k$ is wave number associated with the wavelength, $\omega$ is the angular frequency of the waveform, $t$ is the time and $\phi$ is the initial phase. The phase difference between the electrodes does not 
change with time, which changes in the range from 0 to 360 angular degrees. From a practical point of view, however, since there exist reflected waves from the edges of the 2D sheet, the EM absorber is attached to the sides of $2 \mathrm{D}$ sheet to mitigate the deviation of the RSS at electrodes.

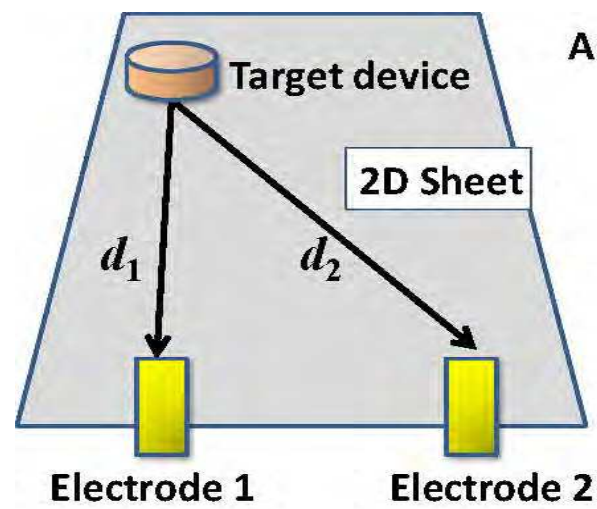

\section{Amplitude (Power)}

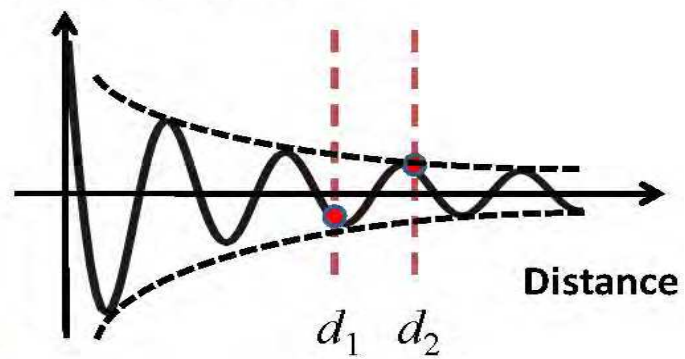

Fig. 8. Difference of received phase and signal strength in the 2D sheet.

Our proposed position identifying method works in two stage processes: the first stage is the database creation stage by preliminary experiment measurement, and the second stage is the position determination stage by comparing the online data with the database of phase difference at each electrode. In the database creation stage, the pilot signal is sent from reference points on the 2D sheet to obtain the mapping database of RSS and phase difference. After constructing the mapping database, the maximum likelihood is utilized to locate device at the online position determination stage.

Because the radio signal in the $2 \mathrm{D}$ communication sheet is less influenced by the outside noises, the RSS captured by the electrode shows a stable distribution with respect to the position of target device. Thus, a likelihood method can be employed to estimate the corresponding positions of target device. However, the inner factors such as reflections at the sides of the sheet or shadows caused by the connectors of the target device influence the propagation of the EM wave to some extent. To confirm such influence level, we have conducted some simulations concerning radio propagation in the $2 \mathrm{D}$ communication sheet where reflections and shadowing effects are taken into account [16]. The results show that the positioning method using the arrival phase difference provide the adequate estimation of location, and the less the reflection coefficient, the better the accuracy. We also evaluate our proposed method by performing actual position measurement on a $30 \mathrm{~cm} \times 30 \mathrm{~cm}$ area, where the phase differences are measured by eight array electrodes corresponding to $2.44 \mathrm{GHz}$ pilot signal. In order to identify the position by sensing the arrival phase difference between any neighboring two electrodes, we first calibrate the phase patterns at all possible positions of target device placed on the top of communication sheet. At each position $\mathrm{p}$, we record an array of multi-receiver output, and memorize them as database. The measuring process is done by a motor driven $\mathrm{X}-\mathrm{Y}$ translator moving to every position on the sheet. The experiment results reveal that our method can detect position at resolution of $2.5 \mathrm{~cm}$ square grid perfectly. 


\section{Digital scope system on the sheet}

\subsection{Scope display for visualizing location-specific contents}

A large and coarse level map is overlaid on the communication sheet or is projected onto it, and the small display device is placed on the sheet. The printed map has no influence on the electromagnetic wave. Because the communication sheet is possible to be made transparent, the coarse map can also be projected from back of the sheet. Hence, the sheet can be attached on screen or table glass as well with the map projected from back or bottom respectively.

The map overlaid on the sheet can be an atlas of world, city, convention site, exhibition room, designed interior space, human body, etc [3]-[5]. The map can also be a table of items as a brief index of categories. Such a map or table can cover a large area or a big collection that a normal PC screen has insufficient resolution to include. In contrast to the map, the underlying media data to display can be selected as those with drastic differences from the map in resolution or type in order to achieve the special effect of the platform. For example, a street view can be displayed according to the position and orientation of the scope display on a road map. Moving the scope display along a street in the map will update the street view in it. This setting can benefit virtual navigation in a large city, which is not flexible on a normal PC window that displays only a limited part of map and the corresponding street view side-by-side. Because the small display of the scope shows details without changing the large map, the user has a good understanding on his/her global location during the exploration of in-depth data.

Another advantage of the scope display is the possibility for visualizing subjects in different modes or media. Figure 9 shows a table for a children's museum on what it becomes when the egg hatched, and how the living species grow up with the scope displays. The map can also be zoomed in on the display and eventually cross different media to visualize a spot, as displayed in Fig. 10. This type of system best fits into a quiz-and-answer diagram and arouses people's curiosity in exploring topics of interest. User can glance over a large category in exposition and then dig into details by placing the display at an interesting spot.

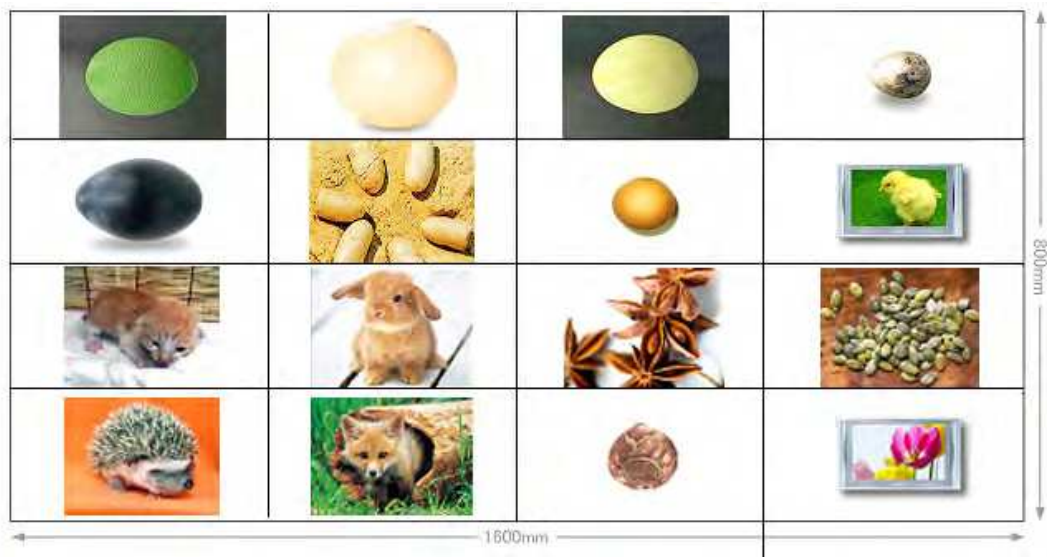

Fig. 9. Scoping details from a category using the communication sheet. 

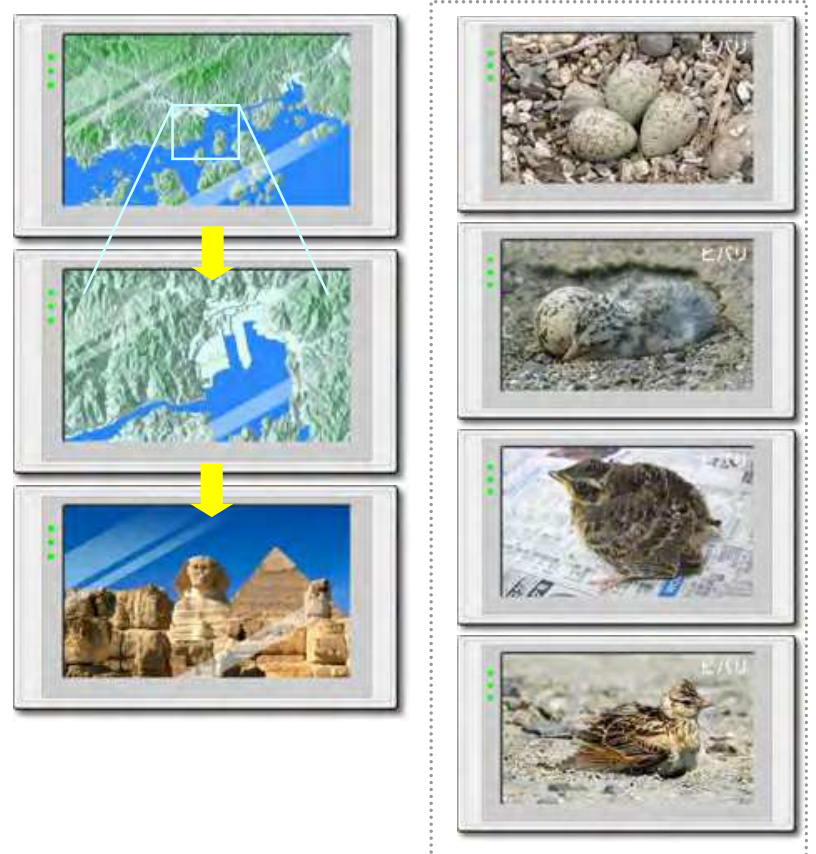

Fig. 10. Zooming in a map across media for location-specific information visualization. (left) from map to scene, (right) how a bird grows up.

\subsection{Data transmission to multi-users}

The system is designed for multi-users, i.e., multiple persons can look at different information at separated locations in the map, which cannot be achieved by a flat screen or touch panel. To meet this goal, multiple media streams have to be sent to the corresponding multiple displays. The design of the platform is depicted in Fig. 11. Here we can employ different channels to send signals to individual displays.

Two approaches are examined here. The first one uses location-free-servers which receive analogue signals from PC output, and convert them to digital signal in IEEE 802.11A $(5.2 \mathrm{GHz})$. In such a framework, the same number of location-free servers as the number of users is needed to send multi-channel signals. Heavy graphics or video contents may even need multiple PCs to render scenes on separated displays. We feed the signals into the sheet through the clip connectors (antenna), and the signals reach everywhere in the sheet for displays to pick up.

The second approach is to use Windows sub-monitors to distribute different contents to displays. Multiple USB LAN output are fed into the sheet by clip connectors and sent to individual displays. The displays that have USB as their input are connected to antenna and is required to register their IDs on the PC first via the communication sheet. This approach requests less resource but can only be used for a few users because of the limitation in the rendering power of a single PC. 


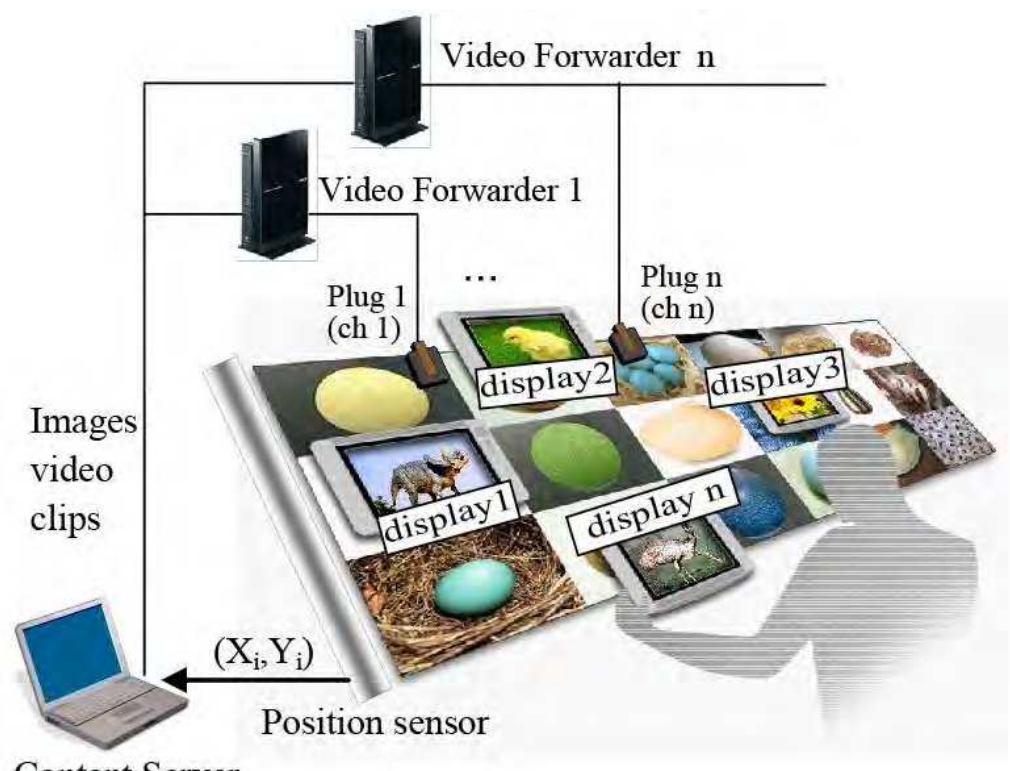

Content Server

Fig. 11. The system design in which the multimedia signals are sent to individual displays for detailed content visualization according to the positions of displays on the communication sheet.

\section{System construction for education and exposition}

The system platform uses a communication sheet originally developed for communication and power supply, which has $2 \mathrm{~mm}$ thickness and a mesh interval of $7 \mathrm{~mm}$. Figure 12 shows power supply via the communication sheet to small devices including speakers, LED lights, monitors, etc. Currently, the communication sheet can be printed at a width of $1 \mathrm{~m}$ and a length of $2 \mathrm{~m}$ for experiments. The cost of such a sheet is low and will be dropped further in mass production for home and office use.

The digital scope display is a Century 4.3 inch screen driven by USB input and the resolution is $800^{*} 480$ pixels. The connector developed is attached to the display from back and it contacts the sheet to pick up signals via the communication sheet. We use a position sensing device reconstructed from MIMIO [17], which has the granularity of 100dpi. A circuit is designed to switch between multiple infrared-ultrasound generators to send out pulses alternatively. The signal reaching the receivers is then sent to computer via USB connection. The position estimation on the computer is programmed with MIMIO SDK. The frequency to compute the position of one generator is $87 \mathrm{~Hz}$, and the Zigbee switching between two positioning sets is $10 \mathrm{~Hz}$.

We have experimented on several types of media contents and demonstrated the functioning system. The contents include a world map with 42 topics in famous sightseeing cities and spots, six categories of living species (41 species ranging from animals to plants) and their growing process exhibited in museum. The system is a new type of interface for users to move on a large map space and peek in detailed information at different locations. 


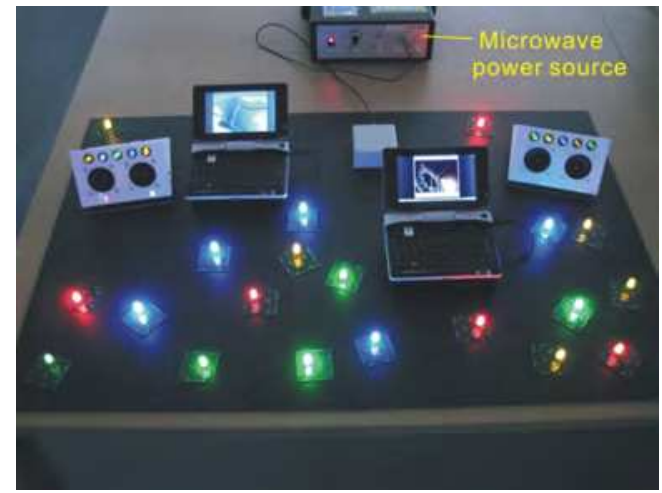

Fig. 12. Power supply via the communication sheet to small devices including speakers, LED lights, monitors, etc.

The large map underlying the small display solves the problem of location/orientation perception in multiwindow system on PCs. For multi-user on the same sheet, we prepare multiple displays connected to a PC for media presentation. The current response time for video clip, however, is a bottleneck that affects the usability of the system. A high performance PC and customized software will be developed to solve this problem.

Based on the estimated positioning data of the display with the reconstructed MIMIO system, the location-specific media details can be magnified and displayed as a digital scope to learning materials, catalogs, tourists guide, X-ray image of building, and various other purposes. For example, as shown in Fig.13, we have developed a digital scope system called

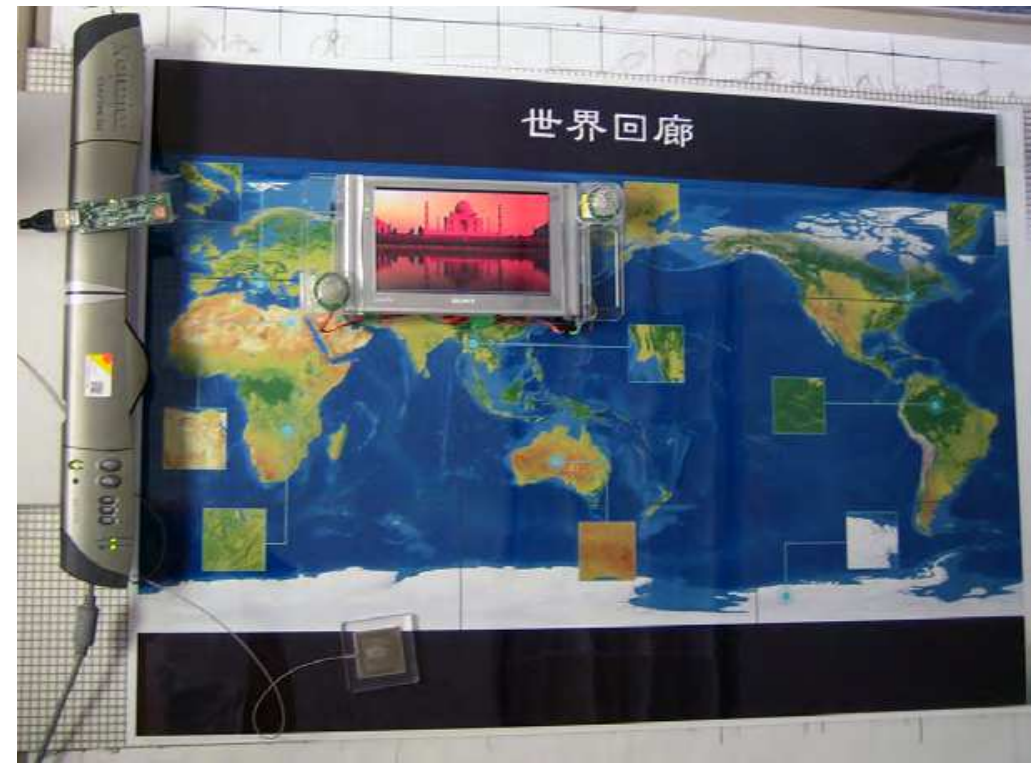

Fig. 13. Digital scope system called "World Corridor". 
"World Corridor." Along the "World Corridor," we can enjoy an around-the-world trip with family and friends while staying at home. Depending on the position where the display is placed, the scenery image of the place on the map can be gradually magnified by zooming in; details of living creatures in the sightseeing spots can also be visualized. We have also developed another digital scope system for children's education materials. Figure 14 shows a picture book of various eggs and species on what it will become when it grows up. For example, as shown in Fig. 14 , when the display is placed in the middle of 2D sheet, the motion pictures are displayed on how the baby chicken were hatched from eggs, grow up and finally develop to adult chicken.

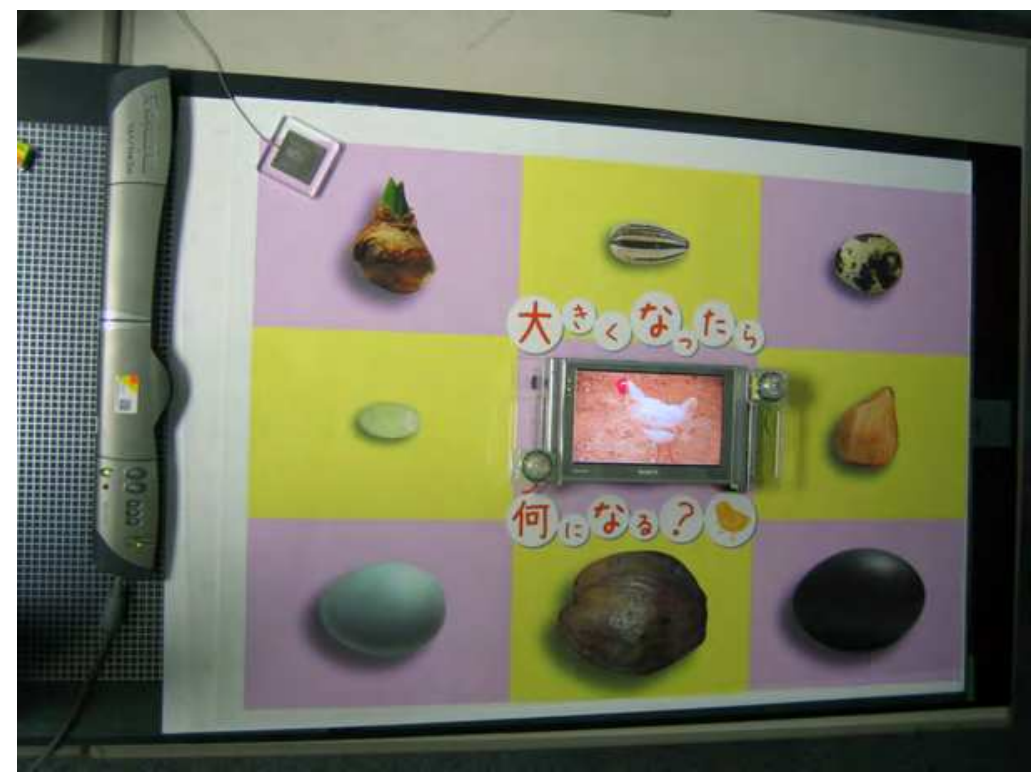

Fig. 14. Digital scope system for children's education materials on what it will become when it grows up.

\section{Discussion and future work}

The proposed digital scope platform is an interface composed of a physical medium and multiple displays to reveal details behind a global map or a general category. It separates the presentation of a map and in-depth data to different media, one on printed paper and others on small screens. This reduces the burden in using a large display or projector in presenting both large map and multiple scope views. A few servers can handle multi-user tasks, which is more flexible than employing a wall of monitors or an array of projectors in conventional setting. It is also energy saving because the signal spread within the communication sheet has less loss than in the air, and in our case only low power signal is sent for communication. The entire system is also convenient to be set anywhere on flat surfaces.

The scope displays are not wired to ensure their free movement on the 2D surface. On the other hand, this platform can send multi-channel signals in the closed sheet by utilizing wider bandwidth than wireless. Multi-channel video can be distributed to different displays 
simultaneously. Moreover, the communication sheet has the location dependent property as compared to wireless in the air; a display placed over different sheets can receive different signals. By partitioning a large space into sheet patches that correspond to regions of interest, a display can receive region-specific data on the patches and will show nothing if it is away from the sheet. This also improves the security in the information access in a large space. The future works include the accuracy improvement of the position sensing on the sheet, which will increase the granularity of the map for dense location information retrieval; lightweight display design for mobility on the sheet; building stereo display with a lenticular sheet for visualizing 3D urban scenes on the map; and adding touching panel on the display to allow viewing different media. We will further consider extending the map to curved surfaces on human body or various objects. For example, partitioned sheets can be wrapped on each part of a manikin or on surfaces of a car to form 3D surface maps. By contacting displays onto surfaces, people will be able to see internal structures, which will help education in engineering and medical areas.

\section{Conclusion}

This chapter describes a multimedia interface platform on the communication sheet named digital scope for visualizing location-specific information. The platform contains small highresolution displays, a large communication sheet, and location sensors. The sensed position of each display is sent to the server and the related media data are transmitted to the display via the communication sheet. Functioning as a scope, users can move the small displays over a large map and look into details at different locations. The multi-channel media data are distributed to facilitate multi-user's interaction. Because of the large map, the locationrelated data are more perceivable in a global layout than in a single screen as current PC that has to present different media at a location side-by-side. The platform is also power efficient than a wall of monitors in achieving the same goal. It will enhance many applications in education and exposition.

\section{References}

[1] J. Y. Zheng, X. Wang, " Pervasive Views: Area exploration and guidance using extended image media ", ACM Multimedia 05, 986-995, 2005. Google Map: http://maps.google.com.

[2] J. Y. Zheng, B. Zhang, H. Cai," Pervasive scene map on wireless devices for city navigation ", NBiS 2009, 75-82.

[3] H. Cai, J. Y. Zheng," Key views for visualizing large spaces ". Journal of Visual Communication and Image Representation 20(6): 420-427 (2009).

[4] J. Y. Zheng, M. Shi, Mapping cityscapes into cyberspace for visualization, J. of Visualization \& Computer Animation, 16(2), 97-107, 2005.

[5] C. Pinhanez, M. Podlaseck, R. Kjeldsen, A. Levas, G. Pingali, N. Sukaviriya, “Ubiquitous interactive displays in a retail environment", SIGGRAPH'03 Sketches. San Diego, California.

[6] M. Podlaseck, C. Pinhanez, N. Alvarado, M. Chan, E. Dejesus, “On interfaces projected onto real-world objects", CHI'03. Florida, 2003 
[7] C. H. Hsiao, L. W. Chan, T. T. Hu, M. C. Chen, J. Hsu, Y. P. Huang, “To move or not to move: a comparison between steerable versus fixed focus region paradigms in multi-resolution tabletop display systems", 153-161, CHI09.

[8] S. Izadi, S. Hodges, S. Taylor, D. Rosenfeld, N. Villar, A. Butler, J. Westhues, "Going beyond the display: a surface technology with an electronically switchable diffuser" UIST 2008: 269-278.

[9] P. Steurer et al., "System design of smart table", IEEE Int. Conf. on Pervasive Computing and Commun. (PerCom), March 2003.

[10] H. Shinoda, "Sensor networking based on two-dimensional signal transmission technology", SICE-ICASE Int. Joint Conf. 2006.

[11] H. Shinoda et al., "Surface sensor network using inductive signal transmission layer," Conf. INSS, pp.201-206, 2007.

[12] Bing Zhang, Azman Osman Lim, Youiti Kado, Hiroto Itai, Hiroyuki Shinoda, "An efficient power supply system using phase control in 2D communication", INSS2009 (Sixth International Conference on Networked Sensing Systems, Pittsburgh, USA), 2009-06.

[13] N. Yamahira, Y. Makino, H. Itai and H. Shinoda," Proximity connection in two dimensional signal transmission", SICE-ICASE Int. Joint Conf., pp.2735-2740, Korea, 2006.

[14] S. Knappe, V. Shah, V. Gerginov, A. Brannon, L. Hollberg, and J. Kitching, "Long-term stability of NIST chip-scale atomic clock physics packages",PTTI2006(38th Annual Precise Time and Time Interval Meeting), 2006-12.

[15] Youiti Kado, Toshifumi Oota, Azman Osman Lim, Bing Zhang, “A simulation study for position estimation of multiple devices in 2D communication system,"in Proc. of the International Symposium on Antennas and Propagation (ISAP), pp.10951098, Bangkok, Thailand, 20-23 Oct. 2009.

[16] MIMIO: http://www.mimio.com 


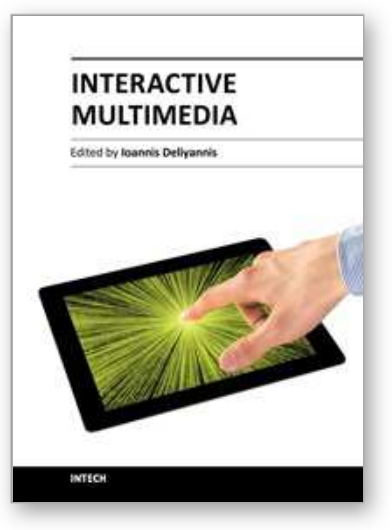

\author{
Interactive Multimedia \\ Edited by Dr loannis Deliyannis
}

ISBN 978-953-51-0224-3

Hard cover, 312 pages

Publisher InTech

Published online 07, March, 2012

Published in print edition March, 2012

Interactive multimedia is clearly a field of fundamental research, social, educational and economical importance, as it combines multiple disciplines for the development of multimedia systems that are capable to sense the environment and dynamically process, edit, adjust or generate new content. For this purpose, ideas, theories, methodologies and inventions are combined in order to form novel applications and systems. This book presents novel scientific research, proven methodologies and interdisciplinary case studies that exhibit advances under Interfaces and Interaction, Interactive Multimedia Learning, Teaching and Competence Diagnosis Systems, Interactive TV, Film and Multimedia Production and Video Processing. The chapters selected for this volume offer new perspectives in terms of strategies, tested practices and solutions that, beyond describing the state-of-the-art, may be utilised as a solid basis for the development of new interactive systems and applications.

\title{
How to reference
}

In order to correctly reference this scholarly work, feel free to copy and paste the following:

Bing Zhang, Youiti Kado, Kiyohiko Hattori and Jiang Yu Zheng (2012). Digital Scope on 2D Communication Sheet for Location-Specific Multimedia Service, Interactive Multimedia, Dr loannis Deliyannis (Ed.), ISBN: 978953-51-0224-3, InTech, Available from: http://www.intechopen.com/books/interactive-multimedia/digital-scopeon-2d-communication-sheet-for-location-specific-multimedia-services

\section{INTECH}

open science | open minds

\author{
InTech Europe \\ University Campus STeP Ri \\ Slavka Krautzeka 83/A \\ 51000 Rijeka, Croatia \\ Phone: +385 (51) 770447 \\ Fax: +385 (51) 686166 \\ www.intechopen.com
}

\author{
InTech China \\ Unit 405, Office Block, Hotel Equatorial Shanghai \\ No.65, Yan An Road (West), Shanghai, 200040, China \\ 中国上海市延安西路65号上海国际贵都大饭店办公楼 405 单元 \\ Phone: +86-21-62489820 \\ Fax: $+86-21-62489821$
}


(C) 2012 The Author(s). Licensee IntechOpen. This is an open access article distributed under the terms of the Creative Commons Attribution 3.0 License, which permits unrestricted use, distribution, and reproduction in any medium, provided the original work is properly cited. 